\title{
X-ray diffraction and molecular-dynamics studies: Structural analysis of phases in diglyceride monolayers
}

\author{
G. H. Peters, ${ }^{1,2}$ N. B. Larsen, ${ }^{3}$ T. Bjørnholm, ${ }^{3}$ S. Toxvaerd, ${ }^{1}$ K. Schaumburg, ${ }^{3}$ and K. Kjaer ${ }^{4}$ \\ ${ }^{1}$ Chemistry Department III, H. C. Ørsted Institutet, University of Copenhagen, Universitetsparken 5, \\ DK-2100 Copenhagen $\emptyset$, Denmark \\ ${ }^{2}$ Novo Nordisk A/S, MedChem Research IV, Novo Nordisk Park, DK-2760 Målbv, Denmark \\ ${ }^{3}$ Centre for Interdisciplinary Studies of Molecular Interactions, Department of Chemistry, \\ University of Copenhagen, Fruebjergvej 3, DK-2100 Copenhagen, Denmark \\ ${ }^{4}$ Physics Department, Risø National Laboratory, DK-4000 Roskilde, Denmark
}

(Received 16 August 1996; revised manuscript received 18 August 1997)

\begin{abstract}
We report a detailed structural analysis of the phases of 1,2-sn-dipalmitoylglycerol Langmuir monolayers at room temperature. Pressure-induced transitions have been investigated by combination of molecular-dynamics simulations and grazing-incidence $\mathrm{x}$-ray diffraction (XRD). The diglyceride film undergoes two phase transitions occurring at 38.3 and $39.8 \AA^{2} /$ molecule. Simulation indicates that the first transition involves a reorientation of the headgroups while simulation and XRD show that in the second transition the order parameter is the tilt angle of the alkyl chains. A methodology for Fourier analysis of simulated Langmuir monolayers is presented. According to the simulation, in the two states of higher surface pressure the alkyl chains are vertical and pack in a centered-rectangular (nearly hexagonal) lattice. In the second phase transition the alkyl chains start tilting. At the lowest pressure the tilt angle reaches $\approx 14^{\circ}$ in a direction close to a nearest neighbor direction. Both arrangements of the alkyl chains are confirmed by XRD. For higher order and fractional order Bragg peaks, simulations predict higher intensities than observed with XRD. This may indicate that in the simulated monolayer the finite size with periodic boundary conditions imposes a higher degree of order. [S1063-651X(98)01503-7]
\end{abstract}

PACS number(s): 68.10.Cr, 61.90.+d, 68.35.Rh, 68.55.Nq

\section{INTRODUCTION}

In recent years considerable progress has been made in refining the application of scanning probe microscopies, imaging, and diffraction techniques [1-4] to study highly ordered molecular films on solid substrates or aqueous subphases. The physical properties of such thin films can be drastically different from those of the corresponding bulk phases. A detailed understanding of the properties of these self-assembled films and the physical origin of the surface structure is fundamentally important to basic and applied problems such as adhesion, capillarity, contact formation, friction, lubrication, wear, modifications of surfaces, etc. [513]. Theoretical approaches [14-16] and, in particular, with the recent developments and implementations of computational methods, simulation techniques [17-28] have been used to elucidate the microscopic origins of these phenomena and their technological consequences.

Some of the most intensively studied systems are lipid monolayers at air-water interfaces (Langmuir films), being particularly attractive model systems for the study of dynamic processes occurring in restricted molecular geometries. Various parameters such as temperature, molecular packing density, molecular composition, and the nature of the subphase can be controlled, and the effect of these variables on, e.g., lateral diffusion can be examined [29]. This is an important aspect in biological membranes, where lateral interactions determine the fluidity and permeability. Fluidity and permeability may modulate the activities of membrane proteins, controlling, e.g., enzyme activity, transport of small molecules across the membrane, or the binding constants of receptors for substances such as hormones, antigens, or nutrients. Most commonly, phase transitions in Langmuir monolayers have been inferred from surface pressure versus molecular area $(\pi-A)$ isotherm measurements [30-32]. A number of new experimental techniques have recently been used to determine structures in monolayers at different length scales. Structures on a mesoscopic scale may be obtained by fluorescence or Brewster angle microscopies [1,3,33-38], whereas structure on a microscopic scale (positional order in, e.g., hexatic and quasicrystalline phases, tilt angle, and orientation of the alkyl chains, rotational order of the backbones of the chains resulting in herringbone or rotator phases and order of ions attracted to the monolayer) can now be deduced from x-ray reflectivity and diffraction methods [39-59] and from neutron reflectivity [60,61]. Information about dynamics, structural orientation of the headgroups at the aqueous subphase, or influence of internal chain conformation on molecular packing and phase stability is, however, experimentally difficult to obtain. Instead, it may be inferred from computer simulation techniques, which complement experimental methods to assess the relative role of order-disorder phenomena involved in lipid monolayer dynamics and phase transitions [62-79]. It has been demonstrated that the simulations reproduce cooperative tilt effects and tilt transitions [66-68] as well as backbone ordering and different rotator phases $[62,63]$. Additional results have been obtained describing headgroup orientation and location of gauche defects in the chains [69-79].

In the present study, we have performed moleculardynamics simulations and a synchrotron x-ray diffraction experiment to determine the structural ordering of 
1,2-sn-dipalmitoylglycerol monolayers at room temperature. Our earlier simulation results indicated that the monolayer undergoes two phase transitions, where the transitions at high and low surface pressures involve the reorientation of the headgroups and tilting of the alkyl chains, respectively. An analysis of the headgroup orientation and distribution of trans-gauche defects in the alkyl chains has appeared $[73,74]$. Here, we present a detailed analysis of the structures observed in the different phases. Though Fourier analysis is often considered in simulations, the structure factors have usually been reported only for a two-dimensional (planar) cut in three-dimensional (3D) reciprocal space. Considering also the third dimension in reciprocal space increases the amount of extractable structural information, such as quantitative descriptions of tilt angle and molecular orientation. Furthermore, higher and fractional order peaks may elucidate the extent of ordering phenomena. In this study, we have calculated both the planar (projected) structure factor intensities and the intensity dependence on all three dimensions of reciprocal space, giving information about the crystalline ordering of the alkyl chains (lattice parameters, tilt, and tilt direction) and the crystalline order of the headgroups. The simulated structures and their calculated structure factors are compared with the x-ray diffraction results.

The paper is organized as follows. In the next section, we briefly present the model and details of the moleculardynamics simulations. In Sec. III we discuss the experimental setup and in Sec. IV we compare the simulation results with the X-ray diffraction data. Finally, in Sec. V, the main findings of our investigation are summarized.

\section{MODEL AND SIMULATION DETAILS}

In the molecular-dynamics calculations, which consist of the integration of the Newtonian equations of motion, we have investigated the dynamical behavior and structural ordering of the diglyceride monolayer at different surface pressures. The monolayers are treated in full atomic detail with the exception that the methyl and methylene units of the alkyl chains of the molecule are modeled as "anisotropic united atoms", (AUA) [74,80]. The different potential functions for the intramolecular contributions (bond length, bond angle, torsion, Lennard-Jones type, and Coulombic energies) and intermolecular interactions (Lennard-Jones type and dipolar energies [81]) have appeared in the literature many times and are fully described in Ref. [74]. The various potential parameters appearing in the potential function are based on recent experimental data or force field calculations and numerical values of the parameters are provided in Ref. [74]. The aqueous subphase is considered to be a continuous medium modeled by an external field [74], whose strength is based on data for the free energy of solvation for simple esters and alcohols. This static field maintains the feature of a soft (flexible) interface and serves as a stabilization of the monolayer.

The simulation was started from one layer of the threedimensional structure of the $L_{\beta}$ mesophase of 1,2-sn-dipalmitoylglycerol [74,82]. 48 chiral molecules were placed in a rectangular simulation cell, where the alkyl chains were perpendicular to the surface and in an all-trans state. Periodic boundaries were imposed in the $x$ and $y$ di- rections and the minimum image convention [83] was employed. The equations of motion were integrated using a leapfrog algorithm [83] with a time step of $t \cong 0.7$ fs [7376]. The temperature was maintained at $25^{\circ} \mathrm{C}$ with a NoseHoover thermostat [84-86]. To obtain a stable film and to avoid mechanical non-equilibrium responses [76], such as strains and stresses within the film, the layer was compressed to a mean molecular area of $A=36.2 \AA^{2} /$ molecule by adjusting the ratio of the simulation cell dimensions such that the off-diagonal pressure tensor components on average equal zero and the diagonal elements equal the spreading pressure [78]. The configuration for the next lower surface density was obtained by expanding the monolayer in the $x$ and $y$ directions while maintaining the ratio of the simulation cell dimensions [87]. Thermodynamic, structural, and dynamic quantities were sampled after thorough equilibration of the film for at least 100 ps. Details of the equilibration and sampling procedure are provided elsewhere [74].

\section{EXPERIMENTAL DETAILS}

Experimental data were obtained using a Langmuir trough for measuring the surface pressure $(\pi)$ versus mean molecular area $(A)$ isotherm and synchrotron x-ray diffraction for determining the packing and conformation of the molecules in the monolayer in situ.

The $(\pi-A)$ isotherm measurements were performed by spreading a $1 \mathrm{mg} / \mathrm{ml}$ solution of (chiral resolved) 1,2-sn-dipalmitoylglycerol (Sigma; $\approx 99 \%$ pure; substantially free of 1,3 isomer) in chloroform (Merck; analytical grade) on Millipore filtered water $(18.2 \mathrm{M} \Omega \mathrm{cm})$ thermostated at $20 \pm 0.2{ }^{\circ} \mathrm{C}$. After evaporation of the solvent, the monolayer was compressed at a rate of $0.4 \AA^{2} /$ molecule min to a surface pressure of $50 \mathrm{mN} / \mathrm{m}$, which is just below the collapse pressure. The compressed monolayer was left for more than $12 \mathrm{~h}$ at constant surface pressure, maintained by adjusting the barrier position, in order to equilibrate. The isotherms were measured with a KSV5000-3 Langmuir trough (KSV Instruments, Helsinki). For comparison with simulation results, experimentally determined isotherms were recorded during the expansion of the compressed monolayer, as discussed in Sec. IV. The expansion rate was $0.03 \AA^{2} /$ molecule min up to a mean molecular area of $45 \AA^{2} /$ molecule, and beyond this point the rate was $0.15 \AA^{2} /$ molecule min. The structure of the Langmuir monolayer was investigated by synchrotron x-ray diffraction using the liquid surface diffractometer on the undulator beamline BW1 at HASYLAB, DESY, Hamburg. A beam of wavelength $1.351 \AA$ was incident on the surface at a shallow angle $\alpha=0.85 \alpha_{c}$ (to enhance surface sensitivity), where $\alpha_{c}$ is the critical angle for total external reflection, giving a footprint of 50 by $5 \mathrm{~mm}$ on the water surface. The background level of scattering was reduced by a He atmosphere inside the trough. Diffracted $x$ rays were detected by a vertically oriented position-sensitive detector with 256 channels mounted behind a Soller collimator, giving a (horizontal) $\left|\vec{q}_{x y}\right|$ resolution (full width at half maximum) of $0.011 \AA^{-1}$ and a (vertical) $q_{z}$ resolution of $0.005 \AA^{-1}$. Here, $\vec{q}=\left(q_{x}, q_{y}, q_{z}\right)$ $=\left(\vec{q}_{x y} ; q_{z}\right)$ is the scattering vector. Since generally Langmuir monolayers are seen to consist of domains that are randomly oriented around the vertical axis, the horizontal com- 


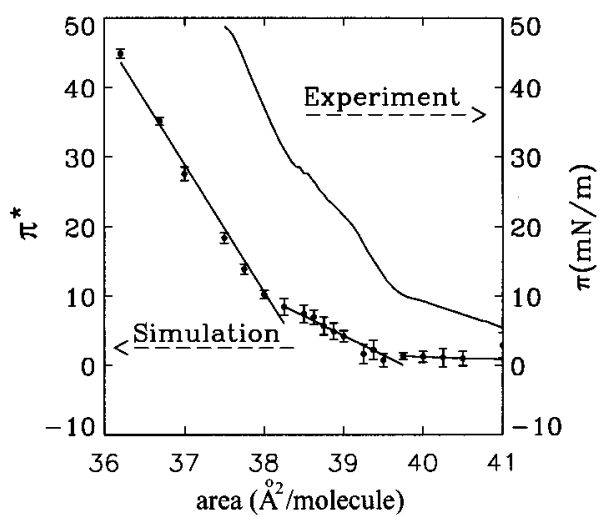

FIG. 1. Surface pressure computed in the simulations (left ordinate) and determined by experiment (right ordinate) during expansion [74]. Computed surface pressures are given in reduced units: $\pi^{*}=0-\gamma \sigma^{2} / \epsilon$, where $\gamma$ is the computed surface tension and $\sigma$ and $\epsilon$ are the Lennard-Jones parameters for methyl groups $(\sigma=3.527 \AA$ and $\epsilon / k_{B}=120 \mathrm{~K} ; k_{B}$ is the Boltzmann constant).

ponents, $q_{x}$ and $q_{y}$, could be resolved only in the combination $q_{x y}=\left|\vec{q}_{x y}\right|=\sqrt{\left(q_{x}^{2}+q_{y}^{2}\right)}$. Spreading conditions were equivalent to those described above. After evaporation of the solvent, the film was compressed at a rate of $1.5 \AA^{2} /$ molecule/min to a surface pressure of $40 \mathrm{mN} / \mathrm{m}$. To allow for equilibration and stabilization of the film, the compressed monolayer was maintained for $1 \mathrm{~h}$ at constant pressure by adjusting the barrier position before the first diffraction scan. Diffraction scans were conducted at constant surface pressures of 40,20 , and $5 \mathrm{mN} / \mathrm{m}$. At the highest surface pressure, a broad scan resolving only $q_{x y}$ was performed to detect the presence of peaks (cf. following section). Peaks were observed only in the range $1.47-1.55 \AA^{-1}$ (corresponding approximately to the interchain distance in a compact hexagonal lattice). Consequently, this range was used for scans simultaneously resolving both $q_{x y}$ and $q_{z}$ at all three surface pressures. In-between scans, lower pressure states were obtained by expanding the film at a rate of $0.1 \AA^{2} /$ molecule $/ \mathrm{min}$.

\section{RESULTS AND DISCUSSION}

Surface tension $\gamma$ and the positional order of the monolayer at different surface pressures are easily calculated in the simulations and can directly be compared with experimental results. In monolayer experiments, the lateral pressure $\pi$ is given by the difference of surface tension in the absence $\left(\gamma_{0}\right)$ and presence $\left(\gamma_{1}\right)$ of amphiphilic molecules at the water surface; $\pi=\gamma_{0}-\gamma_{1}$. In the simulations, the positive spreading pressure $\pi$ is calculated from the intermolecular interactions [88] and the external force field contributions [89].

Surface pressure data are shown in Fig. 1. The experimental isotherm was obtained during expansion. Small hysteresis effects were observed in the isotherms during compressionexpansion cycles (data not shown). The hysteresis is probably caused by large relaxation times of structural ordering in the film. Discontinuities in the slope of the isotherm observed during expansion were not detected upon compression. This is inherent to the diglyceride monolayer film.
Similar observations have been reported, e.g., for long chain aliphatic alcohols $[90,91]$. The isotherms calculated in the simulations and the experimental isotherms measured during expansion of the film show two discontinuities in slope occurring at 38.3 and $39.8 \AA^{2} /$ molecule. Experimentally, the location of the kinks could be determined with a precision of $\pm 1 \AA^{2} /$ molecule, and good agreement is found between simulations and experiment. The differences in the slopes of the isotherms will be discussed later.

Structural information about the simulated Langmuir film was obtained qualitatively by inspection of several snapshots at the different surface pressures [cf. Figs. 2 and 7(c) below], and quantitatively by computing the structure factor intensities. In the simulations, periodic boundary conditions were applied to avoid boundary effects. Hence, the system can be considered as a set of $N_{R}$ replicas (superlattice) of the actual simulation cell. Then, the intensity from the extended system, $I_{\text {ext }}$, is given by [92]

$$
\begin{aligned}
I_{\mathrm{ext}}(\vec{q}) & \propto\left\langle\left|\frac{1}{N_{R}} \sum_{j=1}^{N_{R}} \sum_{i=1}^{N_{P}} \exp \left[i \vec{q} \cdot\left(\vec{R}_{j}+\vec{r}_{i}\right)\right]\right|^{2}\right\rangle \\
& =\left|\frac{1}{N_{R}} \sum_{j=1}^{N_{R}} \exp \left(i \vec{q}_{x y} \cdot \vec{R}_{j}\right)\right|^{2}\left\langle\left|\sum_{i=1}^{N_{P}} \exp \left(i \vec{q} \cdot \vec{r}_{i}\right)\right|^{2}\right\rangle \\
& \equiv I_{\mathrm{Repl}}\left(\vec{q}_{x y}\right) I(\vec{q}),
\end{aligned}
$$

where the two sums are over the different replicas of the system $N_{R}(\rightarrow \infty)$ and over the $N_{P}$ particles in the simulation cell. $\langle\cdots\rangle$ denotes an ensemble average over independent configurations. $\vec{R}_{j}$ is a vector pointing to the origin of the $j$ th replica in the superlattice, and $\vec{r}_{i}$ is the position of the $i$ th particle in the simulation cell. As shown, the double sum can be separated (cf. [93]) into two factors: $I_{\text {Repl }}$ (arising from replication of the simulation cell) gives a set of Bragg peaks corresponding to the superlattice structure: $I_{\text {Repl }}$ is unity for scattering vectors $\vec{q}=\left(\vec{q}_{x y} ; q_{z}\right)$ with horizontal components $q_{x}^{P}=2 \pi \mu / L_{x}$ and $q_{y}^{P}=2 \pi \nu / L_{y}\left(\mu, \nu= \pm 0,1, \ldots ; L_{x}\right.$ and $L_{y}$ are the replication translations in the $x$ and $y$ directions, respectively) while (for $N_{R} \rightarrow \infty$ ) $I_{\text {Repl }}$ (and $I_{\text {ext }}$ ) are zero elsewhere. The spacing between the $\vec{q}_{x y}^{P}$ can only be reduced by increasing the size of the simulation cell. Due to the inherent restriction of system replication along the real space lateral dimensions, any crystalline particle lattice must necessarily be commensurate with the superlattice, so that the reciprocal lattice of the particle lattice will be subset of the $\vec{q}_{x y}^{P}$ lattice. It is conventional in simulation studies to denote as the intensity the factor $I(\vec{q}) \equiv\left\langle\left|\sum_{i=1}^{N_{P}} \exp \left(\overrightarrow{i q} \cdot \overrightarrow{r_{i}}\right)\right|^{2}\right\rangle$, the intensity arising from the $N_{P}$ particles in the simulation cell. As pointed out above, the intensities $I\left(\vec{q}_{x y} ; q_{z}\right)$ are meaningful only for $\vec{q}_{x y} \in\left\{\vec{q}_{x y}^{P}\right\}$ [94].

A top view of the monolayer is shown in Fig. 2. It appears that the chains form a crystalline lattice, where the chains remain straight and parallel [cf. Fig. 7(c) top]. To quantify the ordering of the layer, we have calculated the intensities $I\left(q_{x}^{P}, q_{y}^{P}, q_{z}\right)$ [95]. For simplicity, we first consider projected intensities $\int_{-1}^{1} I\left(q_{x}^{P}, q_{y}^{P}, q_{z}\right) d q_{z}$. A map is shown in Fig. 3, where intensities due to the entire molecules are indicated by the white area of the semicircles and intensities due only to 


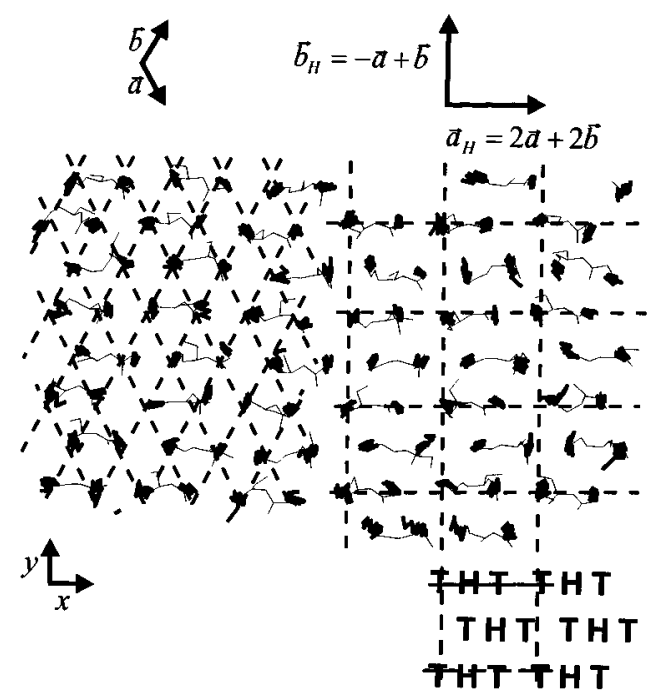

FIG. 2. Top view of a snapshot of the simulation cell at $36.2 \AA^{2}$ (high pressure state). The alkyl chains (drawn in bold lines) form a slightly distorted hexagonal lattice indicated by dashed lines in the left part of the figure. Real space unit vectors $\vec{a}$ and $\vec{b}$ are indicated above the simulation cell. The dashed lines on the right reveal a two-molecule (four-chain) rectangular superlattice, formed by the headgroups, giving rise to fractional order peaks in reciprocal space. The superlattice real space unit vectors $\vec{a}_{H}=2(\vec{a}+\vec{b})$ and $\vec{b}_{H}=-\vec{a}+\vec{b}$ are shown above the cell. The idealized arrangement of heads $(H)$ and alkyl chains $(T)$ is shown below (right).

the headgroups are displayed by the black semicircles (headgroups are indicated by the thin bonds in Figs. 2 and 6). Six dominant peaks are observed in Fig. 3. Using $\vec{a}^{*}$ and $\vec{b}^{*}=(1.394, \mp 0.782) \AA^{-1}$ as the primitive vectors of a reciprocal lattice [97], the six peaks may be written as $\vec{q}_{x y}$ $=h \vec{a}^{*}+k \vec{b}^{*}$ with Miller indices $(h, k)$ as indicated in Fig. 3. For the six dominant peaks the intensities due to the entire molecules are much higher than those due only to the headgroups. We conclude that the six dominant peaks describe the apparently crystalline order of the hydrocarbon chains. From the reciprocal basis vectors $\vec{a}^{*}$ and $\vec{b}^{*}$ we can construct [92] the (nearly hexagonal) real-space lattice
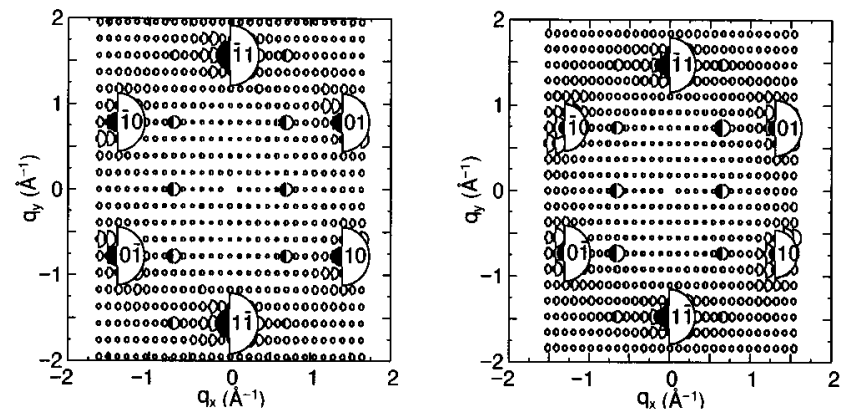

FIG. 3. Calculated projected scattering intensity of the entire molecules (white semicircles) and the headgroups alone (black semicircles) shown for 36.2 (left) and 41.0 (right) $\AA^{2} /$ molecule. The area of the respective semicircles is proportional to the projected intensity $\int_{-1}^{1} I\left(q_{x}^{P}, q_{y}^{P}, q_{z}\right) d q_{z} ; q_{x}^{P}=2 \mu \pi / L_{x}, q_{y}^{P}=2 \nu \pi / L_{y}$. [dashed lines in Fig. 2 (left)] with basis vectors $\vec{a}$ and $\vec{b}$ as shown in Fig. 2 (top left). Projected intensities $\left[\int_{-1}^{1} I\left(h \vec{a}^{*}+\right.\right.$ $\left.k \vec{b}^{*} ; q_{z}\right) d q_{z}$, bars] and Bragg rod intensities [I(h $\vec{a}^{*}$ $+k \vec{b}^{*} ; q_{z}$ ) versus $q_{z}$, dotted lines] are shown in Fig. 4 for a number of different mean molecular areas. To ease the comparison with x-ray diffraction measurements [98], in Fig. 4 we further report intensities (dashed lines) that are calculated as $I^{\text {sum }}(\vec{q})=I\left(+q_{x},+q_{y},+q_{z}\right)+I\left(-q_{x},-q_{y},+q_{z}\right)$. Projected intensities (data not shown) of the $\{11\},\{\overline{1} 2\}$, and $\{2 \overline{1}\}$ peaks, and the $\{02\}\{\overline{2} 2\}$, and $\{20\}$ peaks are $\approx 10 \%$ and $\approx 5 \%$, respectively, of the intensities of the first order peaks $(\{01\},\{1 \overline{1}\}$, and $\{10\})$.

In Fig. 3 intensities of $\approx 5 \%$ of the first order peaks are further observed at the fractional-order positions $\vec{q}_{x y}$ $= \pm\left(+1 / 4 \vec{a}^{*},+1 / 4 \vec{b}^{*}\right), \quad \pm\left(+3 / 4 \vec{a}^{*},-1 / 4 \vec{b}^{*}\right), \quad$ and $\pm\left(+1 / 4 \vec{a}^{*},-3 / 4 \vec{b}^{*}\right)$. These reflections are due to the headgroups (cf. black semicircles in Fig. 3). Their positions may be generated from reciprocal basis vectors $\vec{a}_{H}^{*}=\frac{1}{4}\left(\vec{a}^{*}+\vec{b}^{*}\right)$ and $\vec{b}_{H}^{*}=\frac{1}{2}\left(-\vec{a}^{*}+\vec{b}^{*}\right)$. The resulting [92] real space lattice is shown in Fig. 2 (right): the headgroups form a rectangular superlattice with unit cell vectors $\vec{a}_{H}=2(\vec{a}+\vec{b})$ and $\vec{b}_{H}=$ $-\vec{a}+\vec{b}$ giving rise to the fractional order peaks. The pattern of alkyl chains $(T)$ and headgroups $(H)$ is shown schematically in Fig. 2 (bottom right), and the symmetry of the arrangement results in systematic absences of some of the peaks predicted by the reciprocal basis vectors $\vec{a}_{H}^{*}$ and $\vec{b}_{H}^{*}$ (cf. Fig. 3): All integer-order reflections are present, with contributions from both alkyl chains and headgroups. Only every second row of fractional-order reflections is present, and only the headgroups contribute to those intensities [99]. Projected and $q_{z}$-resolved intensities (Bragg rods) of the headgroups, given in Fig. 5, indicate that there is no major difference between the projected intensities in the most compressed and expanded states. However, differences are observed in the $q_{z}$-resolved intensities.

We now proceed with the analysis of the integer-order peaks. From the Bragg rod profiles shown in Fig. 4 (dotted lines) we have deduced the average orientation of the molecules defined by the tilt angle $\theta$ and the azimuthal angle $\psi$ of the hydrocarbon chains as follows: The features of the intensity distribution along the Bragg rods are determined mainly by the form factor of the aliphatic chains. Now, for nearly parallel long linear aliphatic chains the atom-by-atom structure is largely irrelevant for calculating the intensities and it may be replaced by a smoothed electron distribution resulting in a very slim prolate ellipsoid of length $L$. The form factor of the hydrocarbon chains, being the Fourier transform squared of the electron density, will be a very flat oblate ellipsoid of FWHM thickness $2 \pi / L$ and perpendicular to the chain axis $[96,102]$. Hence, the orientation of the center plane of the oblate ellipsoid (where the form factor is maximum) gives the orientation of the hydrocarbon chains. In Fig. 4, the maxima in the $I\left(h \vec{a}^{*}+k \vec{b}^{*} ; q_{z}\right)$ (dotted lines), at positions $q_{z}=\hat{q}_{z}^{h k}$, result from the molecular form factor. The average orientation of the axes of the hydrocarbon chains can then be determined by least-square fitting, to the points $\left(q_{x}^{10}, q_{y}^{10}, \hat{q}_{z}^{10}\right),\left(q_{x}^{01}, q_{y}^{01}, \hat{q}_{z}^{01}\right),\left(q_{x}^{\overline{11}}, q_{y}^{\overline{1} 1}, \hat{q}_{z}^{\overline{1} 1}\right)$, a plane through the origin, 

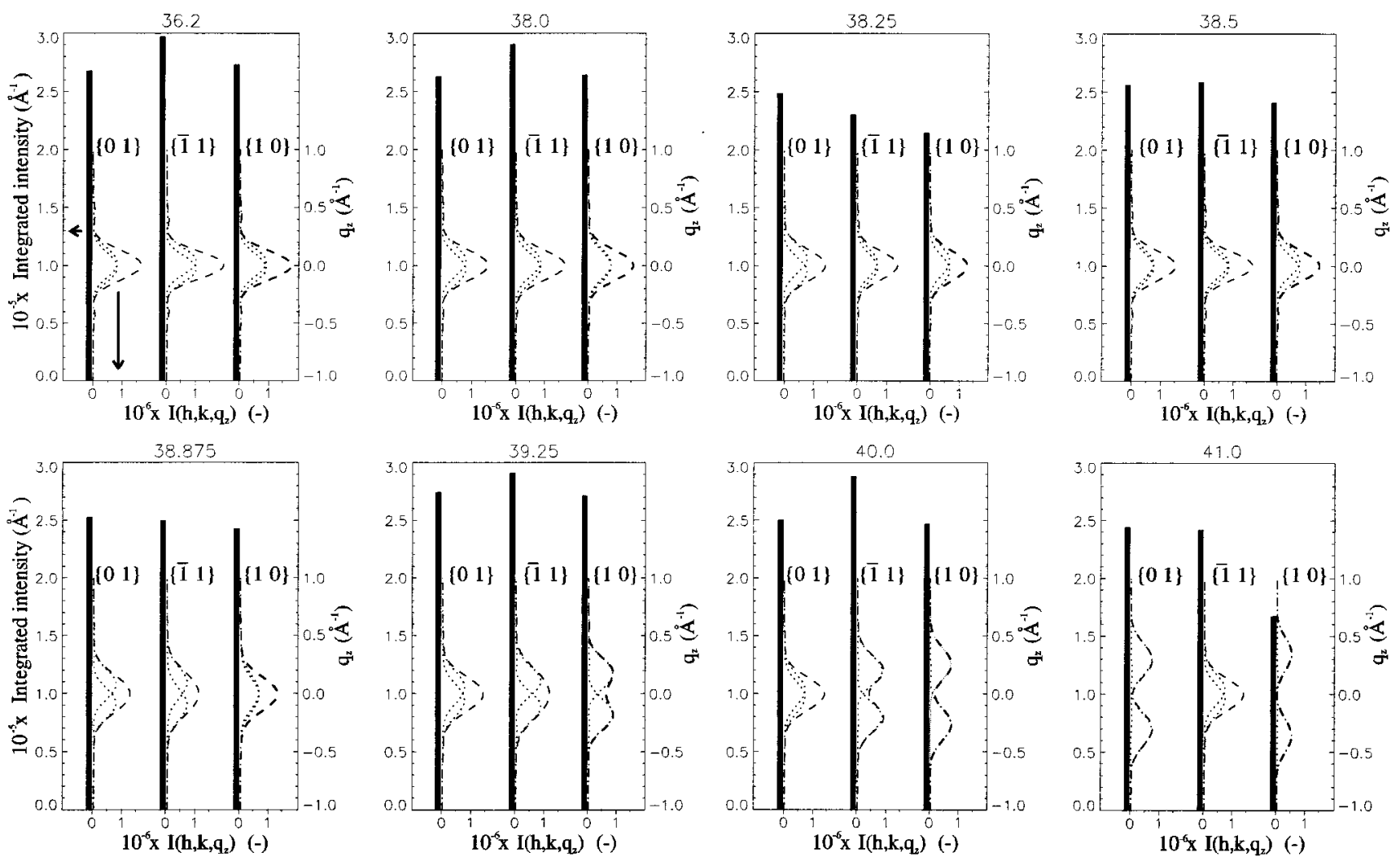

FIG. 4. Diffraction profiles calculated in the simulations at 36.2, 38.0, 38.25, 38.5, 38.875, 39.25, 40.0, and $41.0 \AA^{2} / \mathrm{molecule}$ (as indicated). $\{h k\}$ denotes th $(h k)$ and $(\overline{h k})$ reflections. Dotted curves are $q_{z}$-resolved intensities $\left[I_{h k}\left(q_{z}\right)\right]$ for $-1<q_{z}<1 \AA^{-1}$, where three of these were calculated using the Laue symmetry $\left[I\left(q_{x}, q_{y}, q_{z}\right)=I\left(-q_{x},-q_{y},-q_{z}\right)\right]$ [92]. Dashed lines are the $q_{z}$-resolved summed intensities $\left[I_{\{h k\}}^{\text {sum }}\left(q_{z}\right) \equiv I_{h k}\left(q_{z}\right)+I_{\overline{h k}}\left(q_{z}\right)\right] . I^{\text {sum }}$ is useful because experimentally the intensities $I_{h k}\left(q_{z}\right)$ could not be separated from $I_{h k}\left(q_{z}\right)$. The bars represent projected (i.e., $q_{z}$-integrated) diffraction intensities $I_{\{h k\}}^{\}} \equiv \int_{0}^{1} I_{\{h k\}}^{\text {sum }}\left(q_{z}\right) d q_{z}$ where the integration limits were chosen for comparison with experimental data. Note that by the Laue symmetry $I_{\{h k\}}^{\int}=\int_{-1}^{1} I_{h k}\left(q_{z}\right) d q_{z}$.

$$
\begin{aligned}
0 & =\alpha q_{z}+\beta q_{y}+\gamma q_{z} \\
& \equiv \sin (\theta) \cos (\psi) q_{x}+\sin (\theta) \sin (\psi) q_{y}+\cos (\theta) q_{z} .
\end{aligned}
$$

The chain axis is then orthogonal to the plane, i.e., along the real-space vector $(\alpha, \beta, \gamma)$, having tilt angle $\theta$ and azimuthal angle $\psi$. In Table I, the peak positions $\hat{q}_{z}^{h k}$ and the corresponding peak intensities $I_{h k}\left(\hat{q}_{z}\right)=I\left(h \vec{a}^{*}+k \vec{b}^{*} ; \hat{q}_{z}\right)$ are summarized for different coverages. The orientations of the molecules were then determined from Eq. (2) [4,96,102], resulting in the fitted parameters given in Table II: at a small area per molecule, $A \leqslant 38.5 \AA^{2}$, the alkyl chains are tilted by $1-2^{\circ}$. The low tilt angles, $\theta$ result in an ill-defined azimuthal angle $\psi$. At $40 \AA^{2} /$ molecule, the alkyl chains tilt by $\approx 10^{\circ}$ from the vertical in a lateral direction close to the direction of next nearest neighbor. On further expansion to $41 \AA^{2} /$ molecule, the tilt angle increases to approximately $14^{\circ}$ from the vertical in a lateral direction $\approx 7^{\circ}$ from the $\vec{a}+\vec{b}$ nearest neighbor direction.

While the phase transition at low pressure is due to tilting, the phase transition observed at high surface pressure is not associated with tilting or structural reorientation of the alkyl chains. Simulation results indicate that the transition is caused by a reorganization of the headgroups. A detailed analysis of the headgroup motion [74,76] and its role in biological systems [78] has been presented, and we therefore provide only a brief summary here. At high surface pressure, the alkyl chains are close packed in a hexagonal structure. Due to packing effects, both ester groups cannot simultaneously be in contact with the water phase; if one is down, the other is pushed up into the hydrophobic region and vice versa, as schematically shown by the molecules $(A 1)$ and (A2) in Fig. 6. This cyclic movement can be thought of as a "seesaw mechanism," which is hindered or stabilized by the
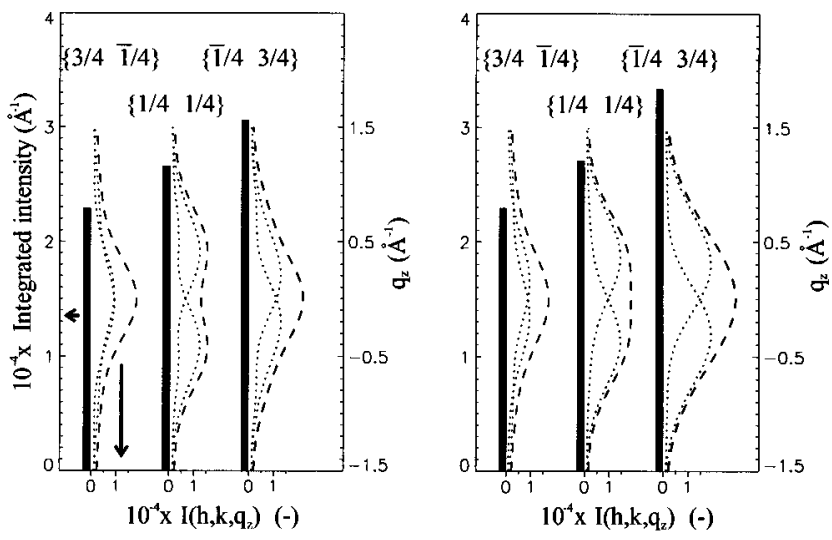

FIG. 5. Diffraction profiles as in Fig. 4 but for the fractionalorder peaks, for 36.2 (left) and 41.0 (right) $\AA^{2} /$ molecule. 
TABLE I. For the Bragg rods, the $q_{z}$ value $\left(\hat{q}_{z}\right)$ giving the maximum intensity is tabulated as well as the maximum intensity $I_{h k}\left(\hat{q}_{z}\right) \cdot q_{z, \text { fit }}$ is the fitted value using Eq. (2). The last column shows the difference between actual and fitted $\hat{q}_{z}$ data.

\begin{tabular}{|c|c|c|c|c|c|c|c|c|}
\hline $\begin{array}{l}A \\
\left(\AA^{2} / \text { molecule }\right)\end{array}$ & $(h k)$ & $\left(\begin{array}{c}q_{x}^{p} \\
\left(\AA^{-1}\right)\end{array}\right.$ & $\left(\AA^{q_{y}^{p}}+1\right)$ & $\begin{array}{c}q_{x y}^{p} \\
\left(\AA^{-1}\right)\end{array}$ & $I_{h k}\left(\hat{q}_{z}\right)$ & $\left(\AA^{\hat{q}_{z}}\right)$ & $\begin{array}{c}q_{z, f i t} \\
\left(\AA^{-1}\right)\end{array}$ & $\begin{array}{c}\hat{q}_{z}-q_{z, f i t} \\
\left(\AA^{-1}\right)\end{array}$ \\
\hline 36.20 & 01 & 1.394 & 0.782 & 1.598 & 825709 & -0.020 & -0.017 & 0.003 \\
\hline 36.20 & $\overline{1} 1$ & 0.000 & 1.565 & 1.565 & 1012197 & -0.030 & -0.033 & -0.003 \\
\hline 36.20 & 10 & 1.394 & -0.782 & 1.598 & 893061 & 0.020 & 0.017 & -0.003 \\
\hline 37.00 & 01 & 1.379 & 0.774 & 1.581 & 845843 & -0.020 & -0.017 & 0.003 \\
\hline 37.00 & $\overline{1} 1$ & 0.000 & 1.548 & 1.548 & 978408 & -0.040 & -0.043 & -0.003 \\
\hline 37.00 & 10 & 1.379 & -0.774 & 1.581 & 788340 & 0.030 & 0.027 & -0.003 \\
\hline 38.00 & 01 & 1.360 & 0.764 & 1.560 & 814053 & -0.040 & -0.040 & 0.000 \\
\hline 38.00 & $\overline{1} 1$ & 0.000 & 1.527 & 1.527 & 866751 & -0.030 & -0.030 & 0.000 \\
\hline 38.00 & 10 & 1.360 & -0.764 & 1.560 & 781291 & -0.010 & -0.010 & 0.000 \\
\hline 38.25 & 01 & 1.356 & 0.761 & 1.555 & 790504 & -0.060 & -0.060 & 0.000 \\
\hline 38.25 & $\overline{1} 1$ & 0.000 & 1.522 & 1.522 & 703557 & -0.020 & -0.020 & 0.000 \\
\hline 38.25 & 10 & 1.356 & -0.761 & 1.555 & 674604 & -0.040 & -0.040 & 0.000 \\
\hline 38.50 & 01 & 1.352 & 0.759 & 1.550 & 764862 & 0.020 & 0.020 & 0.000 \\
\hline 38.50 & $\overline{1} 1$ & 0.000 & 1.517 & 1.517 & 840744 & -0.020 & -0.020 & 0.000 \\
\hline 38.50 & 10 & 1.352 & -0.759 & 1.550 & 765386 & 0.040 & 0.040 & 0.000 \\
\hline 38.88 & 01 & 1.345 & 0.755 & 1.543 & 767758 & -0.080 & -0.080 & 0.000 \\
\hline 38.88 & $\overline{1} 1$ & 0.000 & 1.510 & 1.510 & 687653 & -0.090 & -0.090 & 0.000 \\
\hline 38.88 & 10 & 1.345 & -0.755 & 1.543 & 638988 & 0.010 & 0.010 & 0.000 \\
\hline 39.25 & 01 & 1.339 & 0.751 & 1.535 & 760406 & 0.060 & 0.063 & 0.003 \\
\hline 39.25 & $\overline{1} 1$ & 0.000 & 1.503 & 1.503 & 866345 & -0.110 & -0.113 & -0.003 \\
\hline 39.25 & 10 & 1.339 & -0.751 & 1.535 & 838040 & 0.180 & 0.177 & -0.003 \\
\hline 39.38 & 01 & 1.337 & 0.750 & 1.533 & 747307 & -0.030 & -0.027 & 0.003 \\
\hline 39.38 & $\overline{1} 1$ & 0.000 & 1.500 & 1.500 & 759203 & -0.120 & -0.123 & -0.003 \\
\hline 39.38 & 10 & 1.337 & -0.750 & 1.533 & 728634 & 0.100 & 0.097 & -0.003 \\
\hline 40.00 & 01 & 1.326 & 0.744 & 1.521 & 708864 & 0.030 & 0.040 & 0.010 \\
\hline 40.00 & $\overline{1} 1$ & 0.000 & 1.489 & 1.489 & 848814 & -0.200 & -0.210 & -0.010 \\
\hline 40.00 & 10 & 1.326 & -0.744 & 1.521 & 714239 & 0.260 & 0.250 & -0.010 \\
\hline 41.00 & 01 & 1.310 & 0.735 & 1.502 & 705526 & 0.300 & 0.303 & 0.003 \\
\hline 41.00 & $\overline{1} 1$ & 0.000 & 1.470 & 1.470 & 716408 & -0.040 & -0.043 & -0.003 \\
\hline 41.00 & 10 & 1.310 & -0.735 & 1.502 & 495286 & 0.350 & 0.347 & -0.003 \\
\hline
\end{tabular}

balance of intramolecular and intermolecular forces [74,76]. On expansion, the intermolecular interaction decreases, and at the high pressure transition, the intramolecular interaction between the two chains is strong enough to cause a "swelling" of the molecules [78], allowing the two ester groups to escape from the constrained motion given by the "seesaw mechanism." On further increasing the area per molecule, sufficient space is created that both ester groups can be exposed to the water phase simultaneously as indicated by molecules $(B)$ and $(C)$ in Fig. 6, where conformation $(C)$ represents the tilted state.
In Fig. 7, a subset of the simulation results [corresponding to the three different regions observed in the $(\pi-A)$ isotherm (Fig. 1)] is presented in a form suitable for direct comparison [103] with the XRD data, which are shown in Figs. 8 and 9. Initially, in the high pressure state of the film, a fast scan over a wide range of the horizontal scattering vectors $q_{x y}=\sqrt{\left(q_{x}^{2}+q_{y}^{2}\right)}$ was performed to detect the presence of peaks (while not resolving $q_{z}$ in order to increase speed). Only one peak (due to the alkyl chains) was observed in the range $0.65<q_{x y}<2.87 \AA^{-1}$. Figure 8 shows the measured diffraction profile (bold line). Peaks calculated from 
TABLE II. Fitted parameters used in Eqs. (2) and (2) to describe a plane through the origin and the points $\left(q_{x}^{10}, q_{y}^{10}, \hat{q}_{z}^{10}\right)$, $\left(q_{x}^{01}, q_{y}^{01}, \hat{q}_{z}^{01}\right),\left(q_{x}^{\overline{1} 1}, q_{y}^{\overline{1} 1}, q_{z}^{\overline{1} 1}\right)$. No azimuthal angles $(\psi)$ are given for low tilt angles $(\theta)$, since $\psi$ is ill defined at low $\theta$. The reported errors are standard deviations and were calculated by performing the fitting procedure to each configuration.

\begin{tabular}{crcccc}
\hline \hline$A\left(\AA^{2} /\right.$ molecule $)$ & \multicolumn{1}{c}{$\alpha$} & $\beta$ & $\gamma$ & $\theta$ & $\psi$ \\
\hline 36.20 & 0.000 & 0.021 & 1.000 & $1 \pm 1$ & $n / a$ \\
37.00 & -0.004 & 0.028 & 1.000 & $2 \pm 1$ & $n / a$ \\
38.00 & 0.018 & 0.020 & 1.000 & $2 \pm 1$ & $n / a$ \\
38.25 & 0.037 & 0.013 & 0.999 & $2 \pm 1$ & $n / a$ \\
38.50 & -0.022 & 0.013 & 1.000 & $2 \pm 1$ & $n / a$ \\
38.88 & 0.026 & 0.059 & 0.998 & $4 \pm 2$ & $n / a$ \\
39.25 & -0.089 & 0.075 & 0.993 & $8 \pm 2$ & $140 \pm 21$ \\
39.38 & -0.026 & 0.082 & 0.996 & $6 \pm 2$ & $108 \pm 23$ \\
40.00 & -0.108 & 0.139 & 0.984 & $10 \pm 2$ & $128 \pm 12$ \\
41.00 & -0.241 & 0.029 & 0.970 & $14 \pm 1$ & $173 \pm 6$ \\
\hline \hline
\end{tabular}

the simulations are shown vertically displaced [104]. The two sets of data were put on a common scale by equating the integrated intensities of the first order peaks. The calculated peaks were drawn as Lorentzians with the same FWHM (full width at half maximum) as the measured peaks [106]. The simulation predicts higher order peaks and fractional order peaks that are absent from the XRD data. While the simulated higher order peaks are at the limit of what would be detectable in the XRD, the simulated fractional order peaks are about 7 to 10 times the XRD detection limit.

A detailed scan of the first order peak resolving both $q_{x y}$ and $q_{z}$ showed the Bragg rod to have maximum at the horizon $\left(q_{z} \simeq 0\right)$ in accordance with the simulation results. Simulation also predicts that the headgroup Bragg rods and the higher order alkyl chain Bragg rods should have the major part of their respective intensities at $\left|q_{z}\right|<0.7 \AA^{-1}$. In the fast scan, the range $0<q_{z}<0.8 \AA^{-1}$ was integrated. Due to the absence of other peaks, only the first order alkyl chain

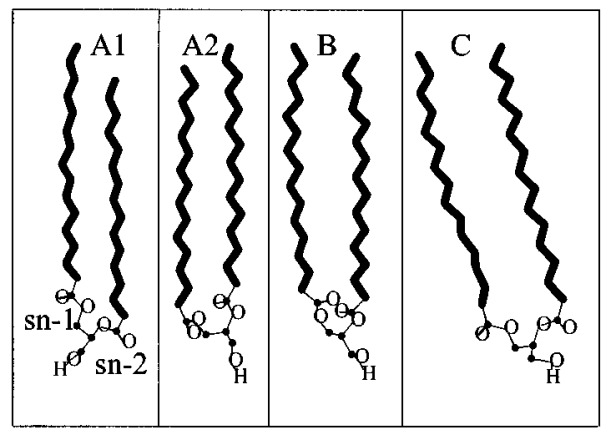

FIG. 6. Conformations observed with high probability density in the simulations at different surface pressures. Structures $(A 1)$ and (A2) show the two limiting arrangements of the sn-1 and sn-2 ester groups at high pressure. Structure $(B)$ is observed at edium pressure, whereas conformation $(C)$ represents the tilted state at low pressure. Hydrocarbon chains are indicated by the thick lines, whereas the headgroup region is indicated by the thin lines. See text for more details.
(A)
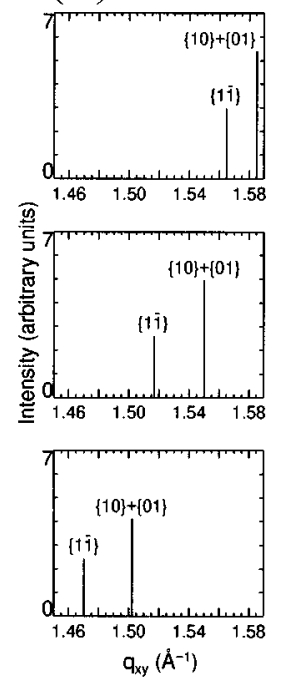

(B)
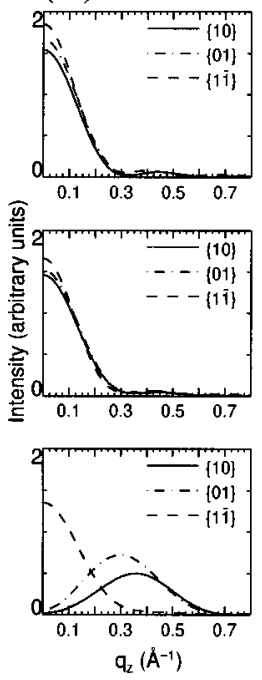

(C)
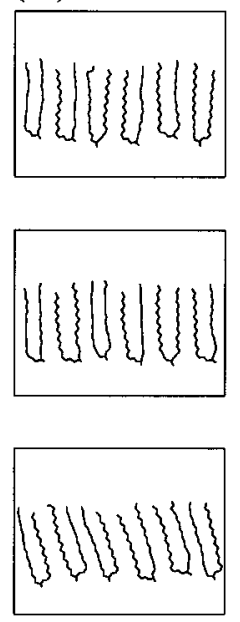

FIG. 7. Diffraction profiles calculated in the simulations at three distinct mean molecular areas (from the top: 37.0, 38.5, and $41.0 \AA^{2} /$ molecule) corresponding to high, medium, and low pressure states. $(A)$ Diffraction peaks: Integrated intensities $I_{\{h k\}}^{j} \equiv \int_{0}^{1} I_{\{h k\}}^{\text {sum }}\left(q_{z}\right) d q_{z}$ as a function of the in-plane scattering vector $\vec{q}_{x y} .\{h k\}$ denotes the sum of $(h k)$ and $(\overline{h k})$ reflections. (B) Intensities $\left[I_{\{h k\}}^{\text {sum }}\left(q_{z}\right) \equiv I_{h k}\left(q_{z}\right)+I_{h k}\left(q_{z}\right)\right]$ summed for equivalent diffraction peaks as a function of the vertical scattering vector component $q_{z}>0$ (Bragg rod profile). (C) Side view: Snapshots of the outermost row of the simulation cell (showing six of 48 molecules) at the three mean molecular areas.

peaks were considered for scans simultaneously resolving $q_{x y}$ and $q_{z}$ at all three investigated surface pressures. The scans resolving $q_{z}$ are presented in Fig. 9. The left column (A) shows contour plots of the measured diffraction intensities depending on both $q_{x y}$ and $q_{z}$. In the center column $(B)$, the scattered intensity is integrated over all PSD channels $\left(0<q_{z}<0.8 \AA^{-1}\right)$, with background subtracted, as a function of horizontal scattering vector $q_{x y}$ component for the three different surface pressures. Diffraction peaks at 40 and $20 \mathrm{mN} / \mathrm{m}$ were fitted to a single Lorentzian, whereas the diffraction peak at $5 \mathrm{mN} / \mathrm{m}$ was fitted by the sum of two Lorentzians. The right column $(C)$ shows the scattered intensity integrated over the $q_{x y}$ range of each peak, with background subtracted, as a function of vertical scattering vector $\left(q_{z}\right)$ component (i.e., Bragg rod scans) [96]. The solid lines represent fits of the Bragg rod scans modeling each alkyl chain as a narrow cylinder of constant electron density and adjusting length, tilt angle, and tilt direction of the cylinder and the surface roughness for the best agreement with the data $[102,96]$. The fitted parameters are given in Table III along with the numerical data for lattice parameters and tilt. From the $q_{x y}$ scans, it was found that the high and medium pressure structures of the alkyl chains are hexagonal. As shown in Fig. 9, at high and medium pressures, the Bragg rod scans are peaked at or close to $q_{z}=0$ corresponding to a collective tilt of the chains $<2^{\circ}[96,102]$. By contrast, the low pressure state shows a distorted-hexagonal phase where the $\{10\}+\{01\}$ peak is displaced in $q_{x y}$ from the $\{\overline{1} 1\}$ peak. The Bragg rod of the $\{10\}+\{01\}$ peak is shifted to higher 


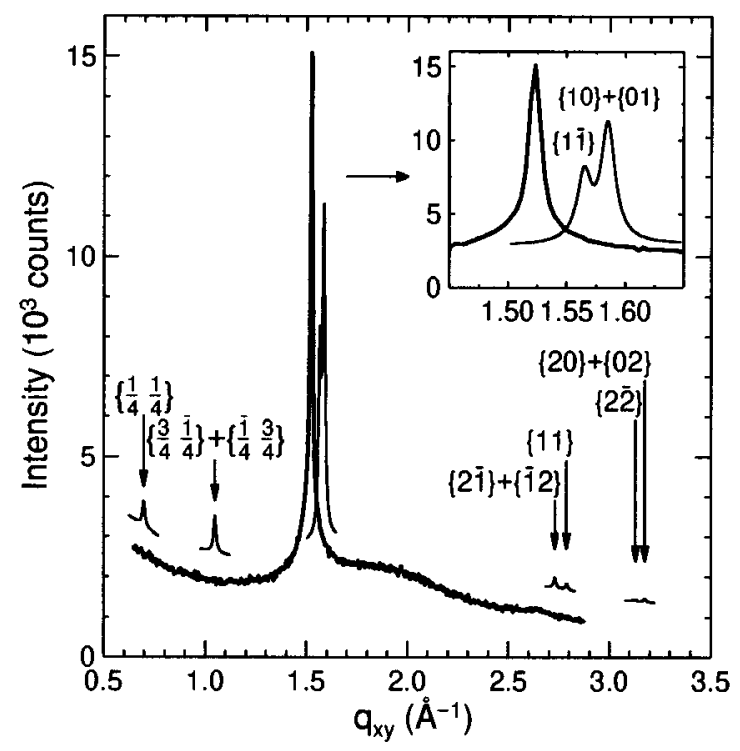

FIG. 8. Experimentally determined diffraction profile (bold line) in the high surface pressure state $(40 \mathrm{mN} / \mathrm{m})$ at a mean molecular area of $38.9 \AA^{2} /$ molecule. The intensity is integrated over all channels of the position sensitive detector as a function of inplane scattering vector $\left|\vec{q}_{x y}\right|$. Diffraction peaks calculated from the simulations (for $A=37.0 \AA^{2}$ per molecule) are shown vertically displaced by 700 counts (LPA and form factor corrected; see text for more detail). The calculated intensities were plotted as peak profiles by normalizing the integrated intensities of the first order peak to the measured peak (after subtracting the background level) and drawing each profile as a Lorentzian with the same width as the measured peak. In addition, the measured background level was added. The inset is a close-up of the region of the first order peaks.

$q_{z}$ values while the $\{\overline{1} 1\}$ Bragg rod remains centered at the horizon $\left(q_{z} \simeq 0\right)$, indicating that the chains are tilted by $14^{\circ}$ in the $\vec{a}+\vec{b}$ direction towards a nearest neighbor chain.

Generally, chirality may lead to packing arrangements in racemic mixtures that are different from those occurring in chiral-resolved compounds. The two-dimensional ordering of phospholipids in Langmuir films is influenced by the headgroup packing resulting in unit cell arrangements of lower symmetry for the chiral compounds than for the racemate [107]. Similarly, it has been observed for phospholipids that chirality plays a key structural and functional role in both cell membranes and plasma lipoproteins [108]. In threedimensional phospholipid crystal structures, molecules are linked by an infinite two-dimensional network of hydrogen bonds involving the hydroxyl groups of the glycerol moiety. In the present study, both in the experiments and in the simulations, 1,2-sn-dipalmitoylglycerol molecules have a chiral carbon center, so that low-symmetrical (oblique) structures might have been expected. However, the dimensions of the headgroups (containing the asymmetric carbon) in the diglyceride molecules are smaller than in phospholipids, and it appears that the packing is predominantly determined by the alkyl chains, resulting in rectangular or higher symmetry.
(A)
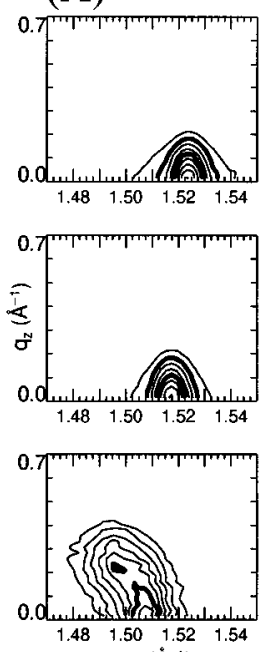

$\mathrm{q}_{\mathrm{xy}}\left(\dot{\mathrm{A}}^{-1}\right)$
(B)
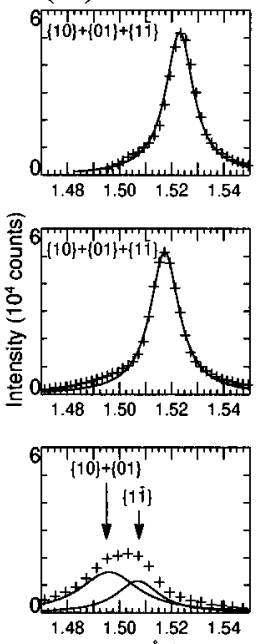

$\mathrm{q}_{\mathrm{xy}}\left(\hat{\AA}^{-1}\right)$
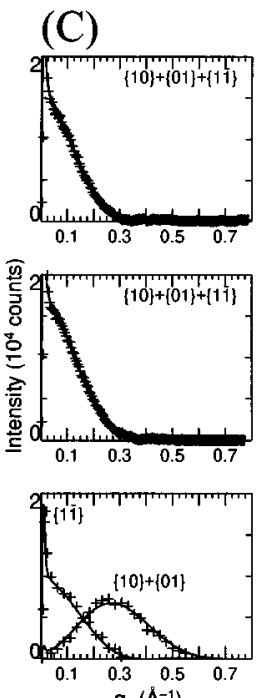

FIG. 9. Experimentally determined diffraction profiles at three mean molecular areas (from the top: 38.9, 39.6, and $40.5 \AA^{2} /$ molecule) corresponding to high $(40 \mathrm{mN} / \mathrm{m})$, medium (20 $\mathrm{mN} / \mathrm{m})$, and low $(5 \mathrm{mN} / \mathrm{m})$ pressure states. $(A)$ Contour plots of the diffraction intensities depending on both in-plane scattering vector $\left|\vec{q}_{x y}\right|$ and vertical scattering vector $\vec{q}_{z}$. In the upper two plots, the contour line spacing is 1000 counts/s (bold contours at 3000 and 6000 counts/s), while the lower plot has a spacing of 100 counts/s and a bold contour at 900 counts/s. (B) Diffraction peaks: intensity integrated over all channels of the detector as a function of the in-plane scattering vector $\left|\vec{q}_{x y}\right|$ (crosses). The solid lines are fits to Lorentzian line shapes. The low pressure state was fitted to two Lorentzians. $(C)$ Bragg rod profiles: diffracted intensity as a function of the vertical scattering vector component $q_{z}$ integrated over the $\left|\vec{q}_{x y}\right|$ range of the corresponding diffraction peak (crosses). The solid lines are fits to a model of the chains as long narrow cylinders. Fitted parameters are given in Table IV and in the text.

\section{CONCLUSION}

In conclusion, we have performed a detailed structural analysis of the phases observed in 1,2-sn-dipalmitoylglycerol Langmuir monolayers based on data from molecular-dynamics simulations and synchrotron x-ray scattering experiments. We have provided the mathematical details of calculating projected and Bragg rod $\left(q_{z}\right.$-resolved $)$ intensities from the simulation data in order to determine structural details such as the orientation of the molecules and for comparison with equally detailed experimental x-ray diffraction data. The simulation results are qualitatively in good agreement with the experimental data, predicting the location of the transitions as well as the absolute value of the molecular tilt angle. We can conclude that the diglyceride film remains in a highly ordered state down to very low surface pressures. At high and medium pressures the alkyl chains pack in a hexagonal structure. The transition at high surface pressure is due to a reorientation of the headgroups as determined by simulation (and not detected in XRD), while the transition at low pressure involves tilting of the alkyl chains by up to $\approx 14^{\circ}$ at the lowest pressure in a direction close to the nearest neighbor direction. The latter transitional reordering is observed by both simulation and x-ray diffraction. The 
TABLE III. Tilt and lattice parameters determined from the $\mathrm{x}$ ray diffraction measurements.

\begin{tabular}{|c|c|c|c|}
\hline Parameters & $40 \mathrm{mN} / \mathrm{m}$ & $20 \mathrm{mN} / \mathrm{m}$ & $5 \mathrm{mN} / \mathrm{m}$ \\
\hline $\begin{array}{l}\text { Surface } \\
\text { roughness }(\AA)\end{array}$ & 2.5 & 2.5 & 2.1 \\
\hline $\begin{array}{l}\text { Length of } \\
\text { alkyl chains }(\AA)\end{array}$ & 18 & 18 & 15 \\
\hline Tilt direction & & & $\vec{a}+\vec{b}$ \\
\hline $\begin{array}{l}\text { Tilt angle of alkyl } \\
\text { chains (degrees) }\end{array}$ & $\leqslant 2$ & $\leqslant 3$ & $\approx 14$ \\
\hline $\begin{array}{l}\text { Tilt azimuthal angle } \\
\text { relative to the }(\vec{a}+\vec{b}) \\
\text { direction (degrees) }\end{array}$ & & & $\leqslant 2$ \\
\hline$d$-spacing $(\AA)$ & & & \\
\hline$\{0,1\},\{1,0\}$ & 4.126 & 4.141 & 4.200 \\
\hline$\{1,-1\}$ & 4.126 & 4.141 & 4.169 \\
\hline $\begin{array}{l}\text { Correlation } \\
\text { length }(\AA)\end{array}$ & 300 & 250 & 80,120 \\
\hline$|\vec{a}|(\AA)$ & $4.76 \pm 0.02$ & $4.78 \pm 0.02$ & $4.83 \pm 0.02$ \\
\hline$|\vec{b}|(\AA)$ & $4.76 \pm 0.02$ & $4.78 \pm 0.02$ & $4.83 \pm 0.02$ \\
\hline$|\vec{a}+\vec{b}|(\AA)$ & $4.76 \pm 0.02$ & $4.78 \pm 0.02$ & $4.86 \pm 0.02$ \\
\hline$\gamma$ (degrees) & 120.0 & 120.0 & 119.5 \\
\hline$A=2 \mathrm{ab} \sin (\gamma)\left(\AA^{2} /\right.$ molecule $)$ & 39.3 & 39.6 & 40.5 \\
\hline
\end{tabular}

simulated system has a higher degree of ordering than observed with XRD in the real system: According to the simulation, the intensities of the $\{11\},\{\overline{1} 2\},\{2 \overline{1}\}$ higher order peaks are at the detection limit of the XRD experiment, while the peaks with fractional indices (which are due to the head groups) are well above the XRD detection limit. Neither higher order peaks nor peaks due to the headgroups were observed in the experiments. The apparently higher ordering of the simulated system is probably due to finite size effects in the simulations, which causes an inherent ordering imposed by the repeated cell. Sample size dependence was also observed in the simulation of simple hydrocarbon monolayers [109]. This study revealed that systems with 64 chains are large enough to obtain statistically meaningful results. The differences observed in the slope of the $(\pi-A)$ isotherms, and lattice parameters (Tables I and III) are probably caused by slightly inaccurate "interaction energy" parameters in the energy functions (e.g., well depth in the Lennard-Jones potential) and/or "excluded volume" parameters (e.g., van der Waals radii) [110]. The influence of these parameters on thermodynamic quantities has been discussed recently [111]. The location of the phase transitions [i.e., the kinks in the $(\pi-A)$ isotherms] and the structural features (i.e., tilt) observed in the simulations are in good agreement with the experimental data indicating that the parameters are adequate for describing the dynamical behavior of the monolayer.

\section{ACKNOWLEDGMENTS}

We thank HASYLAB at DESY, Hamburg, Germany for beam time and the Danish Materials Development program for access to Langmuir-Blodgett equipment (Grant No. 133/ 460-890218). We are indebted to Bernd Struth who kindly helped to keep the XRD experiment running during the night. This work was financially supported by the University of Copenhagen, Novo-Nordisk A/S and the Danish Natural Science Research Council (Grant No. 3.70.01-08/93). Computations were performed at the University of Copenhagen (Grant No. SNF 11-0065-1) and at Novo-Nordisk A/S. G.H.P. would like to acknowledge financial support from the European Biotechnology (Grant No. BIOZ-CT93-5507). N.B.L. acknowledges financial support from the University of Copenhagen.
[1] C. M. Knobler and R. C. Desai, Annu. Rev. Phys. Chem. 43, 207 (1992).

[2] C. M. Knobler, Advances in Chemical Physics, edited by I. Prigogine and S. A. Rice (Wiley, New York, 1990), p. 397.

[3] H. Möhwald, Rep. Prog. Phys. 56, 653 (1993).

[4] J. Als-Nielsen, D. Jacquemain, K. Kjaer, M. Lahav, F. Leveiller, and L. Leiserowitz, Phys. Rep. 246, 251 (1994).

[5] R. G. Horn, J. N. Israelachivili, and F. Pribac, J. Colloid Interface Sci. 115, 480 (1987).

[6] U. Landman, W. D. Luedtke, N. A. Burnham, and R. J. Colton, Science 248, 454 (1990).

[7] J. N. Israelachivili, Intermolecular and Surface Forces (Academic Press, London, 1992).

[8] J. R. Smith, G. Bozzolo, A. Banerjea, and L. Ferrante, Phys. Rev. Lett. 63, 1269 (1989).

[9] M. W. Ribarsky and U. Landman, Phys. Rev. B 38, 9522 (1988)

[10] C. W. Mate, G. M. McClelland, R. Erlandsson, and S. Chiang,
Phys. Rev. Lett. 59, 1942 (1987).

[11] K. K. Han, J. H. Cushman, and D. J. Diestler, Mol. Phys. 79, 537 (1993).

[12] Y. Wang, K. Hill, and J. G. Harris, J. Chem. Phys. 100, 3276 (1994).

[13] S. R. Cohen, G. Neubauer, and G. M. McClelland, J. Vac. Sci. Technol. A 8, 3449 (1990).

[14] G. M. McLelland, Adhesion and Friction (Springer, Berlin, 1989).

[15] S. Sharma and L. V. Woodcock, J. Chem. Soc. Faraday Trans. 87, 2023 (1991).

[16] B. N. J. Persson, Phys. Rev. Lett. 71, 1212 (1993).

[17] P. A. Thompson and M. O. Robbins, Phys. Rev. A 41, 6830 (1990).

[18] S. Y. Liem, D. Brown, and J. H. R. Clarke, Phys. Rev. A 45, 3706 (1992).

[19] T. K. Xia, J. Ouyang, M. W. Ribarsky, and U. Landman, Phys. Rev. Lett. 69, 1967 (1992). 
[20] P. Padilla and S. Toxvaerd, J. Chem. Phys. 101, 1490 (1994).

[21] G. H. Peters and D. J. Tildesley, Phys. Rev. E 52, 1882 (1995).

[22] G. H. Peters and D. J. Tildesley, Phys. Rev. E 54, 5493 (1996).

[23] T. K. Xia and U. Landman, Science 261, 1310 (1993).

[24] G. H. Peters and E. Velasco, Mol. Phys. 84, 1039 (1995).

[25] E. Velasco and G. H. Peters, J. Chem. Phys. 102, 1098 (1995).

[26] F. Y. Hansen, J. C. Newton, and H. Taub, J. Chem. Phys. 98, 4128 (1993).

[27] G. H. Peters and D. J. Tildesley, Langmuir 12, 1557 (1996).

[28] G. H. Peters, Surf. Sci. 347, 169 (1996).

[29] F. Caruso, F. Grieser, P. J. Thistlethwaite, and D. N. Furlong, Macromolecules 27, 77 (1994).

[30] G. Piéroni, Y. Gargouri, L. Sarda, and R. Verger, Adv. Colloid Interface Sci. 32, 341 (1990).

[31] G. Gaines, Insoluble Monolayers at the Liquid-Gas Interface (Interscience, New York, 1966).

[32] A. Ulman, An Introduction to Ultrathin Organic Films (Academic Press, New York, 1991).

[33] G. A. Overbeck and D. J. Möbius, J. Phys. Chem. 97, 7999 (1993).

[34] S. Riviere, S. Henon, J. Meunier, D. K. Schwartz, M.-W. Tsao, and C. M. Knobler, J. Chem. Phys. 101, 10045 (1994).

[35] J. Ruiz-Garcia, T. Qui, H.-W. Tsao, G. Marshall, C. M. Knobler, G. A. Overbeck, and D. J. Möbius, J. Phys. Chem. 97, 6955 (1993).

[36] D. K. Schwartz and C. M. Knobler, J. Phys. Chem. 97, 8849 (1993).

[37] H. M. McConnell, Annu. Rev. Phys. Chem. 42, 171 (1991).

[38] M. Lösche, H. P. Duwe, H. Möhwald, J. Colloid Interface Sci. 126, 432 (1988).

[39] K. Kjaer, J. Als-Nielsen, C. A. Helm, L. A. Laxhuber, and H. Möhwald, Phys. Rev. Lett. 58, 2224 (1987).

[40] C. A. Helm, H. Möhwald, K. Kjaer, and J. Als-Nielsen, Europhys. Lett. 4, 697 (1987).

[41] C. A. Helm, H. Möhwald, K. Kjaer, and J. Als-Nielsen, Biophys. J. 52, 381 (1987).

[42] K. Kjaer et al., Thin Solid Films 159, 17 (1988).

[43] S. Grayer Wolf et al., Science 242, 1286 (1988).

[44] K. Kjaer et al., J. Phys. Chem. 93, 3200 (1989).

[45] D. Jacquemain et al., J. Am. Chem. Soc. 112, 7724 (1990).

[46] B. Lin, M. C. Smith, T. M. Bohanon, G. E. Ice, and P. Dutta, Phys. Rev. Lett. 65, 191 (1990).

[47] R. M. Kenn, C. Böhm, A. M. Bibo, I. R. Peterson, H. Möhwald, J. Als-Nielsen, and K. Kjaer, J. Phys. Chem. 95, 2092 (1991).

[48] A. M. Bibo, C. M. Knobler, and I. R. Peterson, J. Phys. Chem. 95, 5591 (1991).

[49] M. L. Schlossman, D. K. Schwartz, P. S. Pershan, E. H. Kawamoto, G. J. Kellog, and S. Lee, Phys. Rev. Lett. 66, 1599 (1991).

[50] C. A. Helm, P. Tippmann-Krayer, H. Möhwald, J. AlsNielsen, and K. Kjaer, Biophys. J. 60, 1457 (1991).

[51] F. Leveiller et al., Science 252, 1532 (1991).

[52] F. Leveiller et al., J. Phys. Chem. 96, 10380 (1992).

[53] D. K. Schwartz, M. I. Schlossman, and P. S. Pershan, J. Chem. Phys. 96, 2356 (1992).

[54] M. C. Shih, T. M. Bohanon, J. M. Mikrut, P. Zschak, and P. Dutta, Phys. Rev. A 45, 5734 (1992).

[55] J.-L. Wang et al., J. Am. Chem. Soc. 116, 1192 (1994).

[56] F. Leveiller et al., Langmuir 10, 819 (1994).

[57] C. Böhm et al., Langmuir 10, 830 (1994).
[58] M. Li, A. A. Acero, Z. Huang, and S. A. Rice, Nature (London) 367, 151 (1994).

[59] S. P. Weinbach, K. Kjaer, W. G. Bouwman, G. Grübel, J. F. Legrand, J. Als-Nielsen, M. Lahav, and L. Leiserowitz, Science 264, 1566 (1994).

[60] D. Vaknin, K. Kjaer, J. Als-Nielsen, and M. Lösche, Die Makromolekulare Chemie, Macromol. Symp. 46, 383 (1991).

[61] D. Vaknin, K. Kjaer, J. Als-Nielsen, and M. Lösche, Biophys. J. 59, 3125 (1991).

[62] J. Hautman and M. L. Klein, J. Chem. Phys. 91, 4994 (1989).

[63] J. Hautman and M. L. Klein, J. Chem. Phys. 93, 7483 (1990).

[64] J. Harris and S. A. Rice, J. Chem. Phys. 88, 1298 (1988).

[65] M. Milik, A. Kolinski, and J. Skolnick, J. Chem. Phys. 93, 4440 (1990).

[66] P. van der Ploeg and H. J. C. Berendsen, J. Chem. Phys. 76, 3271 (1982).

[67] J. P. Bareman, G. A. Cardini, and M. L. Klein, Phys. Rev. Lett. 60, 2152 (1988).

[68] J. P. Bareman and M. L. Klein, J. Phys. Chem. 94, 5202 (1990).

[69] M. A. Moller, D. J. Tildesley, K. S. Kim, and N. J. Quirke, J. Chem. Phys. 94, 8390 (1991).

[70] S. Karaborni and S. Toxvaerd, J. Chem. Phys. 96, 5505 (1992).

[71] S. Karaborni, Langmuir 9, 1334 (1993).

[72] S. Shin, N. Collazo, and S. A. Rice, J. Chem. Phys. 98, 3469 (1992).

[73] G. H. Peters, S. Toxvaerd, O. H. Olsen, and A. Svendsen, Tens. Det. 30, 264 (1993).

[74] G. H. Peters, S. Toxvaerd, A. Svendsen, and O. H. Olsen, J. Chem. Phys. 100, 5996 (1994).

[75] G. H. Peters, T. Frimurer, S. Toxvaerd, O. H. Olsen, and A. Svendsen, IEEE Comput. Chem. Comput. Biol. 2, 43 (1995).

[76] G. H. Peters, S. Toxvaerd, O. H. Olsen, and A. Svendsen, Langmuir 11, 4072 (1995).

[77] G. H. Peters, S. Toxvaerd, N. B. Larsen, T. Bjørnholm, K. Schaumburg, and K. Kjaer, Nuovo Cimento D 16, 1479 (1994).

[78] G. H. Peters, S. Toxvaerd, N. B. Larsen, T. Bjørnholm, K. Schaumburg, and K. Kjaer, Nature Struct. Biol. 2, 401 (1995).

[79] J. Böcker, M. Schlenkrich, P. Bopp, and J. Brickmann, J. Phys. Chem. 96, 9915 (1992).

[80] S. Toxvaerd, J. Chem. Phys. 93, 4290 (1990).

[81] Lennard-Jones type and dipolar energies were truncated at $2.5 \sigma$ and $L_{y} / 2$, respectively, where $L_{y}$ is the simulation cell dimension in the $y$ direction. At $36.2 \AA^{2} /$ molecule: $L_{x}$ $=15.336 \sigma, L_{y}=9.108 \sigma . \sigma=3.527 \AA$ is the Lennard-Jones parameter for methyl groups.

[82] N. Albon, J. Chem. Phys. 78, 4676 (1983).

[83] M. P. Allen and D. J. Tildesley, Computer Simulation of Liquids (Clarendon, Oxford, 1989).

[84] S. Nose, Mol. Phys. 52, 155 (1984).

[85] W. G. Hoover, Phys. Rev. A 31, 1695 (1985).

[86] S. Nose, J. Chem. Phys. 81, 511 (1984).

[87] At each state point, the pressure tensor components were monitored. The diagonal elements remained equal and the offdiagonal components were on average equal to zero, indicating that during the expansion no shear or anisotropy was imposed on the film.

[88] J. S. Rowlinson and B. Widom, Molecular Theory of Capillarity (Clarendon Press, London, 1984).

[89] S. Toxvaerd, J. Chem. Phys. 74, 1998 (1981). 
[90] J. T. Buontempo and S. A. Rice, J. Chem. Phys. 98, 5835 (1993).

[91] J. T. Buontempo and S. A. Rice, J. Chem. Phys. 99, 7030 (1993)

[92] C. Kittel, Introduction to Solid State Physics (John Wiley, New York, 1971), Chap. 2.

[93] E. Vives and P.-A. Lindgård, Phys. Rev. B 44, 1318 (1991).

[94] Also, for $\vec{q}_{x y} \notin\left\{\vec{q}_{x y}^{P}\right\}, I\left(\vec{q}_{x y} ; q_{z}\right)$ is sensitive to distortion of the simulation cell while maintaining the replication translations, $\vec{L}_{x}$ and $\vec{L}_{y}$.

[95] Intensity along the Bragg rods [96] (i.e. intensity as a function of $q_{z}$ ) was calculated at discrete points separated by $\Delta q_{z}$ $=0.01 \AA^{-1}$, where $q_{z}$ is the component of the scattering vector perpendicular to the water interface. Projected diffraction intensities are integrated over the range $-1<q_{z}<1 \AA^{-1}$.

[96] J. Als-Nielsen and K. Kjaer, in Phase Transitions in Soft Condensed Matter, Vol. B211 of Nato Advanced Studies Institute Series B: Physics, edited by T. Riste and D. Sherrington (Plenum, New York, 1989), pp. 113-137.

[97] Numerical values correspond to Fig. 3 (left).

[98] In the x-ray diffraction measurements only $\left(q_{x y}, q_{z}\right)$ $=\left(\sqrt{\left(q_{x}^{2}+q_{y}^{2}\right)}, q_{z}\right)$ [but not $\left.\left(q_{x}, q_{y}, q_{z}\right)\right]$ could be resolved and negative $q_{z}$ values were inaccessible due to shadowing by the water surface.

[99] We may further describe the head group ordering as follows: The crystalline order of the chains may alternatively be described in terms of a centered-rectangular lattice, $\vec{a}_{r}=\vec{a}+\vec{b}$, $\vec{b}_{r}=-\vec{a}+\vec{b}$, with only even-even and odd-odd reflections of the corresponding reciprocal lattice present. A further cell doubling yields the $\left(\vec{a}_{H}, \vec{b}_{H}\right)$ unit cell, which may be described as a $2 \times \sqrt{3}$ superstructure in Wood's notation [100,101].

[100] E. A. Wood, J. Appl. Phys. 35, 1306 (1964).

[101] F. Jona, J. A. Strozier, Jr., and W. S. Yang, Rep. Prog. Phys. 45, 527 (1982).
[102] K. Kjaer, Physica B 198, 100 (1994).

[103] Since oxygen and methylene have the same number of electrons (8), the unweighted intensities given by Eq. (1) will be proportional to the experimentally determined x-ray diffraction intensities, given by $I_{\exp } \propto\left|\sum_{j=1} f_{j} \exp \left(i \vec{q} \cdot \vec{r}_{j}\right)\right|^{2}$. Here $f_{j}(\sin \theta / \lambda)$ $=f_{j}(q / 4 \pi)$ is the atomic form factor and is, for a given $q$, approximately proportional to the number of electrons.

[104] The measured x-ray intensities are influenced by Lorentz, polarization, and effective area (LPA) factors [4,105], which depend on $q_{x y}$. The calculated intensities used in Fig. 8 have been correspondingly corrected before comparison. Also, for this broad range of $q_{x y}$, the x-ray form factor squared for carbon was included in the calculation.

[105] B. E. Warren, X-ray Diffraction (Addison-Wesley, London, 1969), Chaps. 3 and 4.

[106] The FWHM thus determined could be overestimated if the measured first order peak consists of several partly overlapping narrower peaks, but this can only result in an underestimate of the peak heights when drawing the calculated peaks.

[107] C. Böhm, H. Möhwald, L. Leiserowitz, J. Als-Nielsen, and K. Kjaer, Biophys. J. 64, 553 (1993).

[108] J. A. F. Op Den Kamp, Lipids and Membranes. Past, Present and Future, edited by B. Roelofsen and K. W. A. Wirtz (Elsevier, New York, 1986).

[109] M. Bishop and J. H. R. Clarke, J. Chem. Phys. 95, 540 (1991).

[110] The force determining the packing and dynamics of the monolayer is proportional to $\epsilon$, whereas much stronger dependency is observed for $\sigma$ (force $\propto \sigma^{12}$ ). Due to the strong dependence of the forces on the excluded volume parameters (force $\propto \sigma^{12}$ ) small deviations may have relatively large effects on the packing.

[111] S. Toxvaerd (unpublished). 